\title{
Differential impact of brain damage on the access mode to memory representations: an information theoretic approach
}

\author{
Rosapia Lauro-Grotto, ${ }^{1}$ Elisa Ciaramelli, ${ }^{2}$ Carolina Piccini ${ }^{3}$ and Alessandro Treves ${ }^{4}$ \\ ${ }_{1}^{1}$ Dipartimento di Psicologia Generale, Università degli Studi di Firenze, via San Niccolò 89/a, 50125 Firenze, Italy \\ ${ }^{2}$ Rotman Research Institute, 3560 Bathurst Street, Toronto ON, Canada M6A 2E1 \\ ${ }^{3}$ Dipartimento di Scienze Neurologiche e Psichiatriche, Università degli Studi di Firenze, viale Morgagni 85, 50134 Firenze, Italy \\ ${ }^{4}$ SISSA, Cognitive Neuroscience, via Beirut 2-4, 34013 Trieste, Italy and NTNU, Centre for the Biology of Memory, Trondheim, \\ Norway
}

Keywords: episodic memory, famous faces, hippocampus, human memory, neocortex

\begin{abstract}
Different access modes to information stored in long-term memory can lead to different distributions of errors in classification tasks. We have designed a famous faces memory classification task that allows for the extraction of a measure of metric content, an index of the relevance of semantic cues for classification performance. High levels of metric content are indicative of a relatively preferred semantic access mode, while low levels, and similar correct performance, suggest a preferential episodic access mode. Compared with normal controls, the metric content index was increased in patients with Alzheimer's disease (AD), decreased in patients with herpes simplex encephalitis, and unvaried in patients with insult in the prefrontal cortex. Moreover, the metric content index was found to correlate with a measure of the severity of dementia in patients with AD, and to track the progression of the disease. These results underline the role of the medial-temporal lobes and of the temporal cortex, respectively, for the episodic and semantic routes to memory retrieval. Moreover, they confirm the reliability of information theoretic measures for characterizing the structure of the surviving memory representations in memory-impaired patient populations.
\end{abstract}

\section{Introduction}

Long-term human memory has long been understood not to be a unitary system, but instead to comprise several components, including episodic and semantic memory (Tulving, 2002). It has been proposed that episodic and semantic memory processes make independent contributions to memory retrieval, mediated by distinct neural substrates (e.g. Aggleton \& Brown, 1999; Yonelinas et al., 2005). In the domain of person-related knowledge, for example, the image of Roberto Benigni can either trigger the vivid recollection of having cried after seeing the movie 'La vita è bella', or the mere knowledge of an Italian actor who won some prize at some point, or both.

Neuropsychological studies of brain-injured patients have shown that semantic and episodic memory can be differently affected by cerebral insult. For example, patients with Alzheimer's disease (AD), who show medial-temporal lobe (MTL) damage, may exhibit a 'semantization' of autobiographical memory, being able to provide semantic information about their life but not to re-access the contextual details of past events (Westmacott et al., 2001; Piolino et al., 2003). Similar problems have been observed in patients with lesions in the prefrontal cortex, who may fail to re-experience contextualized personal episodes (Wheeler \& Stuss, 2003) or assemble contextual aspects from different episodes, creating bizarre scenarios (i.e. confabulation, Gilboa, 2004; Ciaramelli \& Ghetti, 2007). This suggests that the MTL and the prefrontal cortex mediate episodic

Correspondence: Dr A. Treves, as above.

E-mail: ale@sissa.it

Received 26 April 2007, revised 6 September 2007, accepted 9 September 2007 access to long-term memory (Levine et al., 2004; Wheeler \& Buckner, 2004; Moscovitch et al., 2006; Eichenbaum, 2006).

Conversely, patients with semantic dementia (Graham \& Hodges, 1997; Lauro-Grotto et al., 1997; Bozeat et al., 2000), a neurodegenerative disorder characterized by bilateral or left-lateralized atrophy of the anterior and inferior temporal neocortex, typically show a severe impairment in knowledge about concepts, facts and people, while retaining semantic information that relates to their personal lives (e.g. the name of their own dog, while ignoring the meaning of the word DOG; Snowden et al., 1994). That is, in contrast to patients with MTL lesions, the memories of patients with more lateral temporal lesions tend to acquire an 'episodic' flavour, consistent with the fact that lateral and inferior temporal cortex are crucial neural correlates of semantic memory.

How can the relative contributions of episodic and semantic access to long-term memory be characterized? One approach was developed by Westmacott \& Moscovitch (2003). They divided famous people who, although equally familiar, were more or less likely to be associated with personal episodic memories into $\mathrm{R}$ names (for 'recollected') and K names (for 'known'). They found that whereas patients with semantic dementia (like normal controls) were faster and more accurate in recognizing $\mathrm{R}$ than $\mathrm{K}$ names, patients with $\mathrm{AD}$ and MTL amnesia did not show such a performance advantage (Westmacott et al., 2004). This is consistent with the notion that the MTL would be critical for accessing (person-related) memories through an episodic route (see also Gilboa et al., 2006). We have proposed a more quantitative approach based on information theoretic measures (Ciaramelli et al., 2006). 


\section{Distinguishing episodic vs semantic memories based on structural differences in their neural substrates}

David Marr (1971) characterized hippocampal networks, connectionally the final component of the MTL memory system, as a simple content-addressable memory, and McNaughton \& Morris (1987) and Rolls (1989) identified with region CA3 the auto-associator that, in Marr's terms, operates retrieval of stored information from an arbitrary partial cue. This is now the mainstream view of how the hippocampus operates in memory, centred on the interpretation of CA3 as a simple attractor neural network (Amit, 1989; Treves \& Rolls, 1994). Importantly, this view regards the representation of new information to be stored in CA3 as equally arbitrary: specialized circuitry involving the dentate gyrus is thought to have evolved, in mammals, precisely in order to allocate to a new event, already represented online as a distributed spatio-temporal pattern of activity of neocortical pyramidal cells, a new, compressed and entirely arbitrary representation expressed by CA3 pyramidal cells (Treves \& Rolls, 1992; Leutgeb et al., 2007).

Thus, any two events, whatever their similarity in content and whatever their degree of overlap in their neocortical representations, will tend to have the same, baseline level of overlap in CA3 (see Leutgeb et al., 2004; Vazdarjanova \& Guzowski, 2004). In this perspective, the information content of a specific event is recalled episodically when its corresponding representation is first retrieved in CA3 by providing a partial cue, activation is then transferred from CA3 to CA1 via the Schaffer collaterals, and from there to the neocortex, where a large distributed pattern of activity is reinstated (Rolls, 2000). The event is then remembered. Crucially, if the cue fails to retrieve the representation of the correct event, it may activate any other representation at random, as their degree of overlap in CA3 is roughly the same.

In contrast to hippocampal memory representations, cortical ones are expressed over a much larger and distributed network of interconnected cortical areas, with a graded variation from modality specific to highly multimodal association areas (Braitenberg \& Shüz, 1991). Each fragment of an event is associated with a stable and nonarbitrary representation within a cortical area, which is re-used for that fragment in diverse contexts. Two events with correlated content are therefore associated in the neocortex to spatio-temporal activity patterns with a corresponding degree of correlation (Kropff \& Treves, 2007). The intrinsic 'compatibility' of memories sharing overlapping anatomical substrates, in contrast to the 'arbitrarity' and sparseness of hippocampal memories, underlies the semantic structure of cortical (as opposed to hippocampal) representations, that is the complex set of links of association, exemplification and abstraction that define their reciprocal connection. Note that the epoch during which such representations were acquired does not enter this distinction, which is therefore neutral as to whether hippocampal involvement, for example in the recognition of famous faces, is limited in time (Bernard et al., 2004).

In this framework, we have developed a task involving the classification of famous faces according to their nationality and field of activity, and reasoned that classifying memory items mainly using semantic cues would lead to a more concentrated distribution of errors, because detected similarities among the set of stimuli should guide performance even in the case of failed recognition. By contrast, in the case of a preferred episodic access, errors should be more randomly distributed, as expected when semantic structures remain unused. We tested the validity of our approach by comparing results in our task with subjective indicators of recollection and familiarity (i.e. $\mathrm{R}$ and $\mathrm{K}$ responses; Tulving, 1985) for the same face stimuli. We found that the frequency of $\mathrm{R}$ responses correlated positively with the level of performance, but negatively with a measure of response concentration that we call metric content (see below), consistent with the idea that in itself the episodic access mode, while supporting performance accuracy, in case of memory failure leaves subjects with limited clues (Ciaramelli et al., 2006).

Here, we apply this approach to different groups of patients, namely patients with $\mathrm{AD}$, which is characterized by a precocious involvement of hippocampal cortices in the neurodegenerative process (e.g. Apostolova et al., 2006; particularly of the CA1 region, Frisoni et al., 2006), patients suffering from herpes simplex encephalitis (HSE), that damages lateral and anterior temporal neocortex in addition to medial-temporal regions (e.g. Kapur et al., 1994), and patients with focal damage in the prefrontal cortex. We expected to find an increase in metric content in patients with $\mathrm{AD}$ compared with healthy individuals, consistent with their documented deficit in accessing memory traces through an episodic route (e.g. Piolino et al., 2003; Westmacott et al., 2004). For the same reason, patients with lesions in the prefrontal cortex should also show an increase in metric content relative to normal controls (Wheeler \& Stuss, 2003). In contrast, because the lateral temporal cortex seems crucial to mediate a semantic access to memory (e.g. Snowden et al., 1994), patients with HSE were expected to show decreased levels of metric content compared with normal controls.

All patients were administered the task twice, with a 10-month delay. If the metric content indeed tracks qualitative changes in memory performance due to neuropathology, then we should find a further increase in this measure in patients with $\mathrm{AD}$ as the disease progresses, and the hippocampal tissue undergoes further deterioration as a result. Moreover, this measure should correlate with standard indexes of the severity of dementia. This trend should not be observed in patients with HSE, who should instead show a decrease in the metric content with time, if anything. In contrast, we do not expect to find time-related changes in metric content in patients with focal prefrontal lesions, because of the non-progressive nature of brain pathology.

\section{Materials and methods \\ Participants}

Three groups of patients took part in the study: (i) a group of patients with $\mathrm{AD}(n=11)$; (ii) a group of patients who had suffered from HSE $(n=6)$; (iii) a group of patients with damage to the frontal lobe (FL; $n=9$ ). In order to assess our novel measures, we took advantage of groups that were participating each in a separate study. Patients were only included, however, who had no other diagnosis likely to affect cognition or interfere with the participation in our study (e.g. significant psychiatric disease, alcohol abuse, etc.). Given that relevant measures in our task depend on age (Ciaramelli et al., 2006), and that age differed significantly across patient groups $\left(F_{2,20}=9.19\right.$; $P<0.001$ ), each patient was matched to two control participants on the basis of gender, age and education, resulting in three control groups of healthy individuals, matched to patients with AD, HSE and FL, respectively (see below).

Normal controls were healthy volunteers who were not taking psychoactive medication, and were free of current or past psychiatric or neurological illness as determined by history. Participants gave their informed consent to participate in the study according to the Declaration of Helsinki (BMJ1991; 302:1194), that was approved by each local Ethical Committee. In order to investigate time-related changes in memory performance due to neuropathology, all patients 
were administered the task twice, with a delay of about 10 months (range $=9-12$ ) between testing sessions. Normal controls were tested only once, given that in a previous study we found no evidence of a change in performance across a 6-month delay in healthy individuals (Ciaramelli, unpublished dissertation).

Tables 1-3 report demographic and clinical data of patients with $\mathrm{AD}, \mathrm{HSE}$ and FL, respectively, as well as the results patients obtained in relevant neuropsychological tests. Neuropsychological tests used differ across patient groups, who were recruited in different laboratories and for other different studies. Unfortunately, neuropsychological results corresponding to the second administration of our task were not available for most of the patients, and are thus not included in the tables.

\section{Patients with AD}

Eleven patients diagnosed with probable $\mathrm{AD}$ participated in the study. Patients were recruited at the Neurology Department of Florence University. Diagnoses were made by a team of neurologists (including author C.P.) based on radiological (SPECT and/or MRI) evidence, clinical and neurological assessment, and performance on neuropsychological tests. They had a mean age of 66.8 years $(\mathrm{SD}=6.2)$, a level of education ranging from 5 to 13 years, and showed lesions involving mainly the MTL. Patients with AD were matched according to gender, age and education to a group of 22 healthy individuals (AD controls) with a mean age of 65.7 years $(\mathrm{SD}=3.8)$ and a mean education of 9.4 years $(\mathrm{SD}=3.1)$. Note that data from our task are analysed for individual subjects, so having more control subjects simply decreases the standard error on mean measures, and allows better estimates of their distributions. Given the limited numbers of patients available in each group, we reasoned that two control subjects per patient was the optimal balance between statistics and time.

The demographic data and neuropsychological scores of patients with $\mathrm{AD}$ are presented in Table 1. Patients underwent the SMID protocol, a standardized Italian battery for the evaluation of the severity of dementia (Bracco et al., 1990). For the general evaluation of dementia we employed the General Dementia Scale index, in the form assuming correction for age and education, which ranges from 0 (absence of dementia) to 6 (very severe dementia). The Mini-Mental State Examination (Folstein et al., 1975) was used for the evaluation of global cognitive efficiency. In order to obtain an estimate of semantic deterioration, we used the Set Test (Isaacs \& Kennie, 1973), which assesses verbal fluency for four different semantic categories with $40 \mathrm{~s}$ as time limit, and a maximum score of 40/40. Finally, in order to estimate episodic memory deficits, we included the Remote Memory Test (see Bracco et al., 1990) and the Short Story Test, a prose-recall test that had been standardized for the Italian population by Bracco et al. (1990). Individual results obtained in our task were correlated with these standard scores.

\section{Patients with HSE}

Six patients suffering from HSE participated in the study. Patients were recruited at the San Bortolo Hospital in Vicenza, Italy, with the collaboration of Dr Francesca Borgo. All had a diagnosis of meningo-encephalitis of probable herpetic origin, based on clinical as well as instrumental evidence, conducted in the preceding 4 years. After the analysis of the cerebrospinal fluid with polymerase chain reaction assays, which was positive for HSE virus, all patients were treated with Acyclovir. Patients with HSE had a mean age of 50 years $(\mathrm{SD}=9.4)$, and presented lesions involving the anterior

TABLE 1. Demographic and clinical data of the patients with $\mathrm{AD}$

\begin{tabular}{|c|c|c|c|c|c|c|c|c|}
\hline \multirow[b]{2}{*}{ Patients with AD } & \multirow[b]{2}{*}{ Sex } & \multirow[b]{2}{*}{ Age (years) } & \multirow[b]{2}{*}{ Education (years) } & \multirow[b]{2}{*}{ MMSE } & \multirow[b]{2}{*}{ GDS } & \multicolumn{3}{|l|}{ SMID Battery } \\
\hline & & & & & & Remote Memory Test & SET Test & Short Story Test \\
\hline $\mathrm{BI}$ & $\mathrm{F}$ & 56 & 13 & 10 & 6 & 1 & 21 & 0 \\
\hline $\mathrm{TL}$ & $\mathrm{F}$ & 62 & 5 & 13 & 4 & 6 & 40 & 0 \\
\hline MS & $\mathrm{F}$ & 66 & 12 & 22 & 4 & 6 & 40 & 2 \\
\hline $\mathrm{CM}$ & $\mathrm{F}$ & 66 & 10 & 14 & 5 & 2 & 29 & 0 \\
\hline $\mathrm{CV}$ & $\mathrm{F}$ & 72 & 5 & 20 & 5 & 1 & 30 & 4 \\
\hline GL & $\mathrm{F}$ & 78 & 3 & 24 & 3 & 3 & 32 & 8 \\
\hline $\mathrm{FG}$ & $\mathrm{M}$ & 60 & 3 & 27 & 3 & 5 & 31 & 0 \\
\hline $\mathrm{BP}$ & $\mathrm{M}$ & 61 & 5 & 26 & 3 & 4 & 39 & 5 \\
\hline PG & M & 66 & 10 & 18 & 4 & 6 & 36 & 3 \\
\hline FD & M & 67 & 8 & 16 & 5 & 3 & 21 & 1 \\
\hline $\mathrm{BF}$ & M & 67 & 5 & 20 & 5 & 2 & 26 & 0 \\
\hline
\end{tabular}

AD, Alzheimer's disease; F, female; GDS, General Dementia Scale; M, male; MMSE, Mini Mental State Examination.

TABLE 2. Demographic and clinical data of the patients with HSE

Patients with HSE Sex Age (years) Education (years) Raven Progressive Matrices (Coloured) Word Span Letter Fluency Category Fluency Prose Recall

\begin{tabular}{|c|c|c|c|c|c|c|c|c|}
\hline BAI & $\mathrm{F}$ & 50 & 5 & 18 & $2.8^{*}$ & 51 & $17^{*}$ & $9.75^{*}$ \\
\hline MIO & $\mathrm{F}$ & 75 & 5 & 41 & 3.5 & 24.4 & 12 & $1.25^{*}$ \\
\hline MU & $\mathrm{M}$ & 30 & 13 & 31 & 4.5 & $2.8^{*}$ & $3.5^{*}$ & $2^{*}$ \\
\hline BAR & $\mathrm{M}$ & 49 & 5 & 36 & $2.75^{*}$ & $26.3^{*}$ & $11.5^{*}$ & $5.75^{*}$ \\
\hline SAR & $\mathrm{M}$ & 56 & 5 & 30 & 4 & 24.8 & 57 & 8.1 \\
\hline CAL & $\mathrm{M}$ & 44 & 13 & - & - & - & - & - \\
\hline
\end{tabular}

F, female; HSE, herpes simplex encephalitis; M, male. *Impaired performance. 
TABLE 3. Demographic and clinical data of the patients with FL

\begin{tabular}{|c|c|c|c|c|c|c|c|c|c|c|c|}
\hline $\begin{array}{l}\text { Patients } \\
\text { with FL }\end{array}$ & $\begin{array}{l}\text { Sex } \\
\text { (years) }\end{array}$ & $\begin{array}{l}\text { Age } \\
\text { (years) }\end{array}$ & Education & Aetiology & $\begin{array}{l}\text { Description of } \\
\text { lesion }\end{array}$ & MMSE & Stroop Test & WCST & Letter Fluency & Category Fluency & Prose Recall \\
\hline $\mathrm{CC}$ & $\mathrm{F}$ & 44 & 13 & $\mathrm{ACoA}$ aneurysm & Right vmPFC & 27 & $29 *$ & 9 & 31 & 57 & 11 \\
\hline $\mathrm{EF}$ & $\mathrm{F}$ & 42 & 10 & ACoA aneurysm & Bilateral vmPFC & 24 & $58^{*}$ & $66^{*}$ & 35 & 42 & $5^{*}$ \\
\hline VM & $\mathrm{F}$ & 70 & 8 & $\mathrm{ACoA}$ aneurysm & Left vmPFC & 24 & $47^{*}$ & $42 *$ & 18 & 43 & $4 *$ \\
\hline ML & $\mathrm{F}$ & 61 & 8 & $\mathrm{ACoA}$ aneurysm & Left vmPFC & 24 & $52 *$ & 24 & 22 & 32 & 14 \\
\hline $\mathrm{RR}$ & $\mathrm{F}$ & 40 & 14 & ACoA aneurysm & Bilateral vmPFC & $22 *$ & 27 & $31 *$ & $17 *$ & $23^{*}$ & $3^{*}$ \\
\hline LG & $\mathrm{M}$ & 55 & 8 & ACoA aneurysm & Right vmPFC & 25 & 27 & 9 & $13^{*}$ & 26 & $5 *$ \\
\hline PA & $\mathrm{F}$ & 34 & 14 & TBI & Bilateral OFC & 27 & 25 & 9 & 33 & 46 & 5 \\
\hline MP & $\mathrm{M}$ & 25 & 13 & TBI & Right fronto-temporal & 24 & $40 *$ & $25^{*}$ & 25 & 38 & 6 \\
\hline SU & $\mathrm{M}$ & 35 & 18 & TBI & Left fronto-temporal & 30 & 18 & 9 & 35 & $21^{*}$ & 7 \\
\hline
\end{tabular}

ACoA, anterior communicating artery; F, female; M, male; MMSE, Mini Mental State Examination; OFC, orbitofrontal cortex; TBI, traumatic brain injury; vmPFC, ventromedial prefrontal cortex; WCST, Wisconsin Card Sorting Test. *Impaired performance.

and lateral temporal lobe in addition to more medial pathological changes. Five of them have been described in detail elsewhere (see Borgo \& Shallice, 2001). Each patient was matched to two control participants on the basis of gender and age. The control group, involving 12 healthy individuals (HSE controls), had a mean age of 50 years $(\mathrm{SD}=8.7)$ and a mean education of 8 years (range: $5-13)$. Patients with HSE were administered the Raven Progressive Coloured Matrices, measures of Category and Letter Fluency, the word-span test, and the Babcock Prose Recall test (see Spinnler \& Tognoni, 1987 for normative data). Unfortunately, neuropsychological data for one of the patients with HSE were not available. Patients showed preserved reasoning abilities at the Raven Progressive Matrices. However, they were generally impaired in tests of letter and category fluency. Moreover, most of them showed impaired memory performance.

\section{Patients with FL}

Nine patients with damage to the FL participated in the study. Patients were recruited at the Center for Studies and Research in Cognitive Neuroscience, Cesena. They were selected by a neurologist on the basis of the location of their lesion evident on CT or MRI scans. In six out of the nine cases, lesions were the result of the rupture of an aneurysm of the anterior communicating artery, and mainly involved the ventromedial prefrontal cortex (BAs 10, 12, 32, 24). The other three patients had sustained traumatic brain injury, and had lesions involving the orbitofrontal cortex, the lateral prefrontal cortex and the temporal lobe. In view of this heterogeneity, data analysis was repeated, restricted to the six patients with aneurysm alone. Overall, patients with FL had a mean age of 45.1 years $(\mathrm{SD}=13)$ and a mean of 12 years of education $(\mathrm{SD}=3.2)$.

Patients with FL were matched to a group of 18 healthy individuals (FL controls) with a mean age of 44 years $(\mathrm{SD}=8.4)$ and a mean education of 12 years $(\mathrm{SD}=3.1)$. The demographic and neuropsychological profile of patients with FL is presented in Table 2. Patients with FL were administered a battery of neuropsychological tasks aimed at assessing memory and executive functions, involving the MMSE, the Wisconsin Card Sorting Test, The Stroop Test, The Tower of London Test, measures of Category and Letter Fluency, and the Babcock Prose Recall test (see Spinnler \& Tognoni, 1987 for normative data). With the exception of patient RR, patients with FL showed preserved general cognitive abilities at the MMSE. However, they were impaired in tests of executive function. In particular, five out of the nine patients showed abnormal interference effects in the Stroop
Task, and four of them showed a high proportion of perseverative errors in the Wisconsin Card Sorting Test. Memory performance was impaired in four of the patients.

\section{The Famous Faces Multiple Choice Test (FFMCT)}

The FFMCT requires subjects to classify a set of 54 pictures of famous people into $S=9$ disjoint categories according to nationality (Italian, Other European and American) and field of activity (Sportspeople, Politicians, Actors and Singers), as shown in Fig. 1. The picture categories represent the nine combinations of nationality by field of activity. Each category includes six famous faces from across the 20th century, two of whom became famous roughly in the 1940-1950s, two in the 1960-1970s, and two in the 1980-1990s (see Ciaramelli et al., 2006 for the full list of characters).

\section{Procedure}

Subjects were tested individually. Subjects sat at a table, with the FFMCT in front of them. The deck of pictures was placed on the desk, and participants were instructed to pick up one picture at time and place it in one of the nine boxes, the one they felt the most appropriate. Participants were encouraged to take their time to make the decision but, even though the order of pictures in the deck was randomly determined for each participant, they were not allowed to reinsert 'difficult' cards in the deck in order to deal with them later. The experimenter was not allowed to provide any help or feedback and, in case of failed recognition, subjects were simply instructed to guess. No time limit was given for the performance, which usually lasted between 15 and $35 \mathrm{~min}$.

\section{Relevant measures}

Performance accuracy: mutual information and percent correct

The performance of each subject can be described by a matrix $Q\left(s, s^{\prime}\right)$, i.e. the 'Confusion Matrix', reporting the fraction of all cards for which the image belonged to category $s$ and was classified by the subject as $s^{\prime}$. A first summary measure of performance is obviously percent correct classification, which can be expressed as the cumulative fraction distributed along the diagonal, $f_{\text {corr }}=\Sigma_{s} Q(s, s)$. A second summary measure of the correspondence between the actual categories and those that were assigned by the subject is mutual information, 

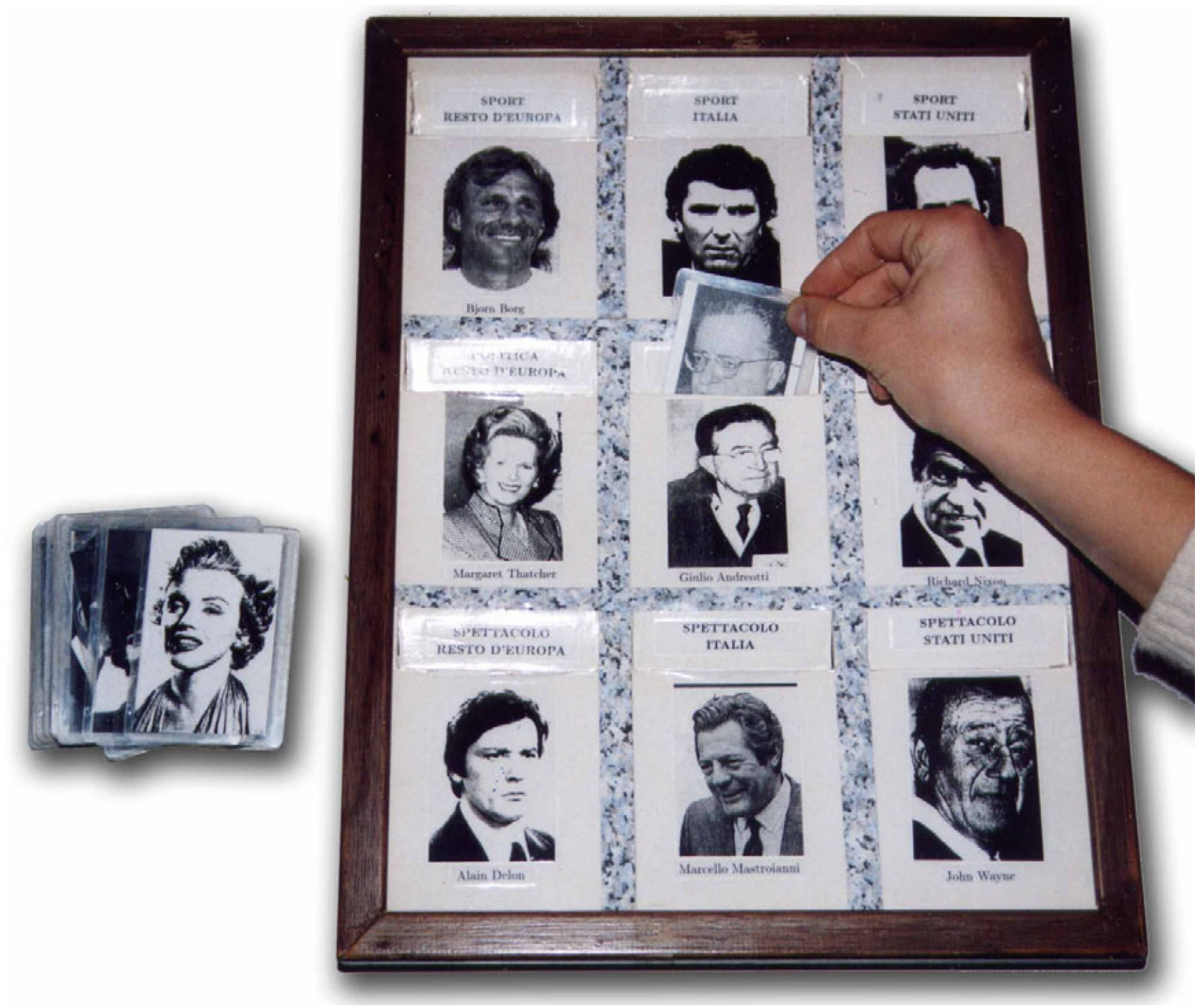

FIG. 1. The FFMCT requires subjects to 'post' a deck of 54 cards, each into the mail-box labelled with the most appropriate category. The nine characters on each mail-box are examples, not included in the deck.

$$
I=\sum_{s, s^{\prime}} Q\left(s, s^{\prime}\right) \log _{2}\left[Q\left(s, s^{\prime}\right) / P(s) Q\left(s^{\prime}\right)\right]-C_{1}
$$

where $P(s)=1 / 9$ is the a priori fraction of cards in each category, $Q\left(s^{\prime}\right)$ is the fraction of responses in category $s^{\prime}$ (cumulated over the actual category of each picture), and $C_{1}$ is a correction term that removes most of the bias due to using fractions (i.e. relative frequencies) rather than probabilities [see Panzeri \& Treves, 1996; note that the bias is subtracted to make the estimate of $I$ mathematically correct (Carlton, 1969), but as $C_{1}$ is constant across subjects, it does not affect the results of statistical tests].

\section{Performance accuracy across decades: the diachronic index}

In order to examine potential differences in performance associated with names that were famous in a remote compared with a recent past, we computed a 'diachronic index', $D$, as the difference between percent correct performance on characters famous mainly in the 19401950 s and on characters famous mainly in the 1980-1990s. Note that in our previous study involving a large sample of healthy participants varying in age (Ciaramelli et al., 2006), we found that no Pearson correlation between $D$ and any socio-demographic variable was significant, except for the obvious one, age.

\section{Concentration of errors: metric content index and partial errors}

In the FFMCT, a misclassified picture can still be assigned to the correct nationality or to the correct field of activity; furthermore, it is possible that a subject shows idiosyncratic classification patterns, such as a tendency to confuse solely 'American Politicians' with 'Europeans Politicians', or else solely among 'Americans', 'Politicians' with 'Actors and Singers'. That is, errors can be entirely random or they can be clustered according to the nature of some (incomplete) semantic cueing.

For a given $f_{\text {corr, }}$, a theoretical minimum value of $I$ can be derived, corresponding to the case of random distribution of incorrect responses 
(Treves, 1997). Setting for simplicity $Q\left(s, s^{\prime} \neq s\right)=\left(1-f_{\text {corr }}\right) /(S-1)$, as if it were a matrix of probabilities rather than relative frequencies (an approximation that becomes exact for decks with many cards),

$$
I_{\text {min }}=\log _{2} S+f_{\text {corr }} \log _{2} f_{\text {corr }}+\left(1-f_{\text {corr }}\right) \log _{2}\left[\left(1-f_{\text {corr }}\right) /(S-1)\right] .
$$

The theoretical absolute maximum of $I$ for a given $f_{\text {corr }}$, on the other hand, is obtained when all incorrect responses are grouped in a single category $s^{\prime}$ (different for each correct category $s$ ), in which case $I=\log _{2} S+f_{\text {corr }} \log _{2} f_{\text {corr }}+\left(1-f_{\text {corr }}\right) \log _{2}\left(1-f_{\text {corr }}\right)$. This maximum, however, would only be produced by a systematic misclassification. A more sensible reference value can be derived by assuming unbiased classification (incorrect categories can at most be chosen as frequently as the correct one) and, for the sake of mathematical simplicity, by assuming that the number of categories is a real (not an integer) number. Under these conditions, the maximum of $I$ corresponds to the case in which all pictures are categorized in clusters of size $1 / f_{\text {corr }}$ and, at each trial, the cluster is correctly identified but the category inside it is selected at random (Treves, 1997), in which case

$$
I_{\max }=\log _{2} S+\log _{2} f_{\text {corr }} .
$$

Thus, when producing relatively low values of $I$, close to $I_{\min }$, the subject behaves as if not perceiving any particular pattern of similarity among the famous people in the pictures, other than among those in the very same category. In this case, the classification, when incorrect, is random. On the contrary, when $I$ is relatively high around the reference value $I_{\max }$, the subject can detect similarities among some of the famous people in the data set, and therefore errors in classification tend to be more clustered, producing an increase in the mutual information value of the classification for a given $f_{\text {corr }}$. Intermediate situations can be conveniently characterized by quantifying the relative amount of information for a given $f_{\text {corr }}$ with the index $\lambda=\left(I-I_{\min }\right) /\left(I_{\max }-I_{\min }\right)$.

Such metric content index varies between 0 and approximately 1 , quantifying the degree to which relationships of being 'close' or 'distant' among stimuli have been relevant to their perception and classification (Treves, 1997). For $\lambda \sim 0$ such relationships are irrelevant, and if a stimulus is misclassified the probability of assigning it to any of the wrong categories is the same. For $\lambda \sim 1$, categories can be thought of as clustering into an arbitrary but systematic semantic structure, while the particular category within each cluster is chosen at random. The metric content index is therefore an estimate of the amount of structure embedded in the neural representations that inform subject choice: it is high when individual memory items are classified using semantic cues, which leads to a more clustered distribution of errors; it is low when episodic access to the identity of each famous face is prevalent, semantic relationships remain largely unused and errors are therefore randomly distributed.

The metric content measure can be conveniently visualized by plotting $I$ as a function of $f_{\text {corr }}$, with the additional lines indicating $I_{\min }\left(f_{\text {corr }}\right)$ and $I_{\max }\left(f_{\text {corr }}\right)$ (which converge for $f_{\text {corr }}=1 / S$ and for $f_{\text {corr }}=1$, giving the diagram the appearance of a leaf, see Fig. 2). The relative vertical excursion of a data point between these two lines represents the metric content of the classification produced by a given subject. This kind of representation is particularly suitable to compare and analyse the performance of groups of subjects at the FFMCT, in that quantitative differences in the joint $f_{\text {corr }}-\lambda$ distribution are reflected in the different positions they occupy in the 'leaf' diagram.

It is worth noting that, given the nature of the metric content estimate, any tendency towards systematic misclassification, not only

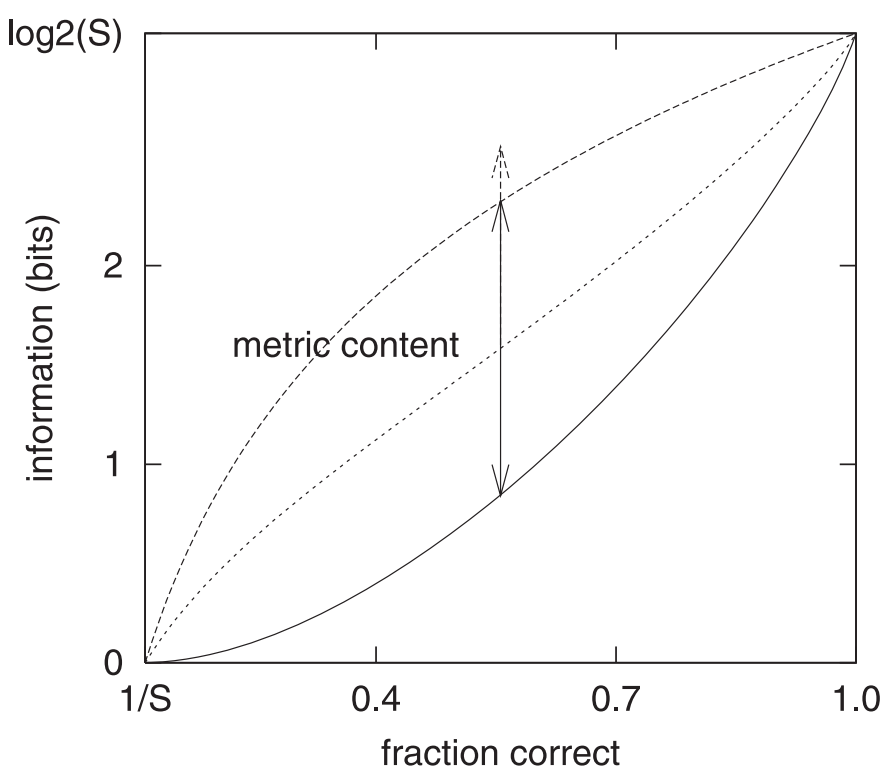

FIG. 2. The 'leaf diagram', where the metric content index ë parametrizes the range of possible mutual information values from $I_{\min }\left(f_{\text {corr }}\right)$ (solid curve) up to and sometimes beyond $I_{\max }\left(f_{\text {corr }}\right)$ (long-dashed curve) for a given fraction of correct responses. The short-dashed curve corresponds to $\lambda=0.5$.

the correct identification of super-ordinates discussed next, would result in an increase of $\lambda$.

A particular case of clustering that might be relevant to this task is super-ordinate categorization: accessing some semantic properties of the pictures might allow to classify, for example, an unrecognized Italian actor in one of the 'Actors and Singers' rather than the 'Sportspeople' categories, while missing his correct Italian nationality. This misclassification would qualify as a partial error, i.e. a classification error in which either of the two super-ordinate classes (nationality or field of activity) is chosen correctly, as opposed to an error in which the chosen class was not correct in terms of nationality or of field of activity (double error). Thus, the 'quality' of misclassification in the FFMCT might in principle be described by distinguishing between partial and double errors (see e.g. Seidenberg et al., 2002). A high frequency of partial errors would indicate that access to the super-ordinate categories is relatively preserved, with the ability to make knowledge-driven classification decisions. Such a measure, however, is likely to reflect not only semantic knowledge, but also a capacity to put that knowledge to use when making classification decisions. In contrast, given that the metric content reflects the amount of structure emerging from the concentration of both correct and incorrect choices in clusters that may emerge irrespective of the six super-ordinate categories available on the FFMCT, we hypothesize that executive functions might have a lower impact on metric content than they have on the proportion of partial errors. In order to compare results concerning metric content and partial errors, we adopted the fraction of partial errors on total errors, $f_{\text {p.e. }} / f_{\text {err }}$ (where $f_{\text {err }} \mathrm{O}_{1}-f_{\text {corr }}$ ) as a measure of their relative frequency, which we will more simply refer to as the proportion of partial errors from now on.

\section{Results}

\section{Patients with $A D$}

Patients with $\mathrm{AD}$ showed a lower mean mutual information, I (1.62 vs 2.07; Student's $t_{31}=-3.6, P<0.001$ ) and a lower mean percent 
correct, $f_{\text {corr }}\left(0.40\right.$ vs 0.64 ; Student's $\left.t_{31}=-6, P<0.0001\right)$ than did their control subjects. Mean values for $I$ and $f_{\text {corr }}$ remained almost unvaried from the first to the second testing session $(P>0.05$ in both cases), when the patients with AD still showed severely impaired performance ( $I$ : 1.62 vs 2.07 ; Student's $t_{31}=-3.7, P<0.001 ; f_{\text {corr }}$ : 0.38 vs 0.64 ; Student's $t_{31}=-7, P<0.0001$; see Fig. $3 A$ ). Patients were impaired in memory for famous faces from remote compared with recent past comparably to controls, as we did not find any difference in the diachronic index, $D$, either at the first or the second testing session $(P>0.05$ in both cases; see Fig. 3B, and summary in Table 4).

In contrast, the metric content, $\lambda$, was significantly higher in patients with $\mathrm{AD}$ compared with controls at the first testing session ( 0.80 vs 0.66 ; Student's $t_{31}=2.2, P<0.05$ ). Further, $\lambda$ tended to increase from the first to the second session in patients with AD $(0.80$ vs $0.90 ; P>0.05)$, resulting in an even more pronounced difference between patients with $\mathrm{AD}$ and normal controls at the second testing session ( 0.90 vs 0.66 , Student's $\left.t_{31}=-3.5, P<0.001\right)$. Interestingly, the proportion of partial errors, $f_{\text {p.e. }} / f_{\text {err }}$, was similar between patients with $\mathrm{AD}$ and controls across sessions (first session: 0.72 vs 0.71 ; second session: 0.69 vs $0.71, P>0.05$ in both cases).

As Fig. 3A shows, while all the control subjects and seven of the patients with $\mathrm{AD}$ are placed in the median area of the leaf diagram, four patients are placed above the upper reference value $\lambda=1$.

In order to elucidate the relation between metric content and changes in long-term memory associated with the neurodegeneration typical of AD, we correlated the scores patients attained in the SMID protocol (see Table 1) with the indices obtained at the FFMCT by the patients with $\mathrm{AD}$ at the first testing session. None of the former correlated with $I, f_{\text {corr }}$ or $f_{\text {p.e. }} / f_{\text {err }}$. In contrast, $\lambda$ was correlated with the GCDC index of severity of dementia $\left(r_{\text {Pearson }}=0.61, P<0.05\right)$, and with performance in the Remote Memory test, although in this case without reaching statistical significance $\left(r_{\text {Pearson }}=-0.45\right.$, $P=0.1)$. Interestingly, $\lambda$ was significantly anti-correlated with the semantic fluency measure $\left(r_{\text {Pearson }}=-0.66, P<0.05\right)$. No correlation was observed between metric content and results in the MMSE or the Short Story Test $(P>0.05)$.

\section{Patients with HSE}

As for the patients with HSE (Fig. 4), in the first testing session they showed lower $I$ (1.63 vs 2.28; Student's $\left.t_{16}=-2.6, P<0.05\right)$ and $f_{\text {corr }}\left(0.53\right.$ vs 0.70 ; Student's $\left.t_{16}=-2.5, P<0.05\right)$ than did normal controls. Similar to the patients with AD, patients with HSE got worse in the second compared with the first session, and differences in

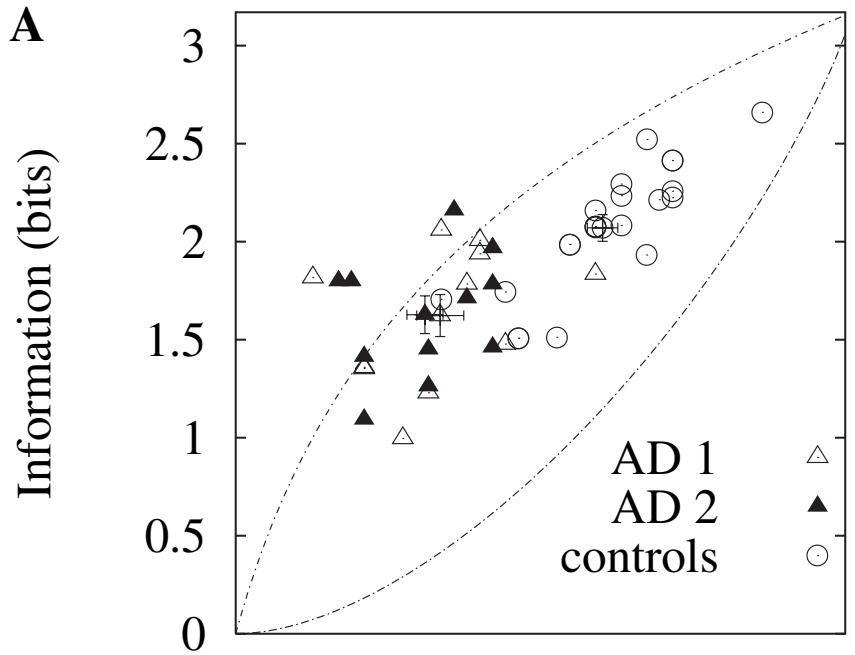

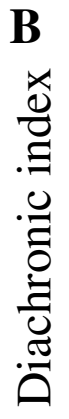

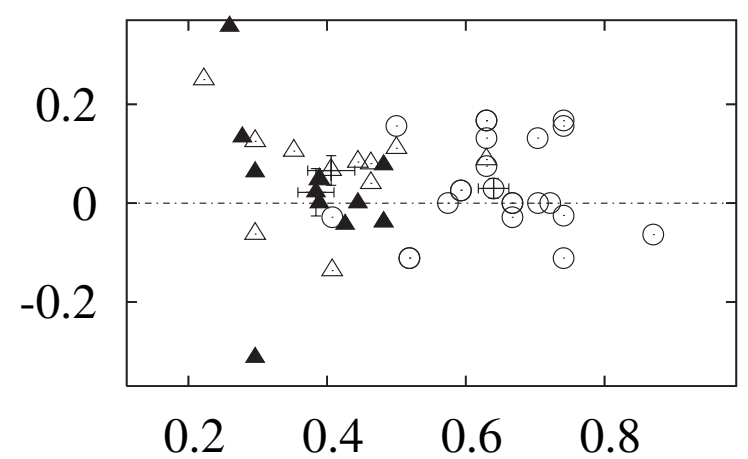

Fraction correct

FIG. 3. Performance of patients with Alzheimer's disease (AD) and of their controls. Scatterplots of mutual information vs fraction correct, as in the leaf diagram (A, top), and of diachronic index vs fraction correct (B, bottom). Group averages are reported with the same symbols as individual data, but with added error bars $( \pm \mathrm{SEM})$. Some individual data points are exactly superimposed.

accuracy between patients and controls were larger at the second session ( $I$ : 1.27 vs 2.28 ; Student's $t_{16}=-2.6, P<0.05$; $f_{\text {corr: }}: 0.44$ vs 0.70 ; Student's $\left.t_{16}=-3.5, P<0.005\right)$. The patients' memory impairment was comparable for recent and remote famous faces.

TABLE 4. Group averages for FFMCT measures

\begin{tabular}{llllr}
\hline & $f_{\text {corr }}$ & $I$ & $\lambda$ & $D$ \\
\hline AD I & $0.40 \pm 0.03^{*}$ & $1.62 \pm 0.11^{*}$ & $0.80 \pm 0.06^{*}$ & $f_{\text {p.e. }} / f_{\text {err }}$ \\
AD II & $0.38 \pm 0.03^{*}$ & $1.62 \pm 0.09^{*}$ & $0.90 \pm 0.07^{*}$ & $0.06 \pm 0.03$ \\
Contr $\times$ AD & $0.64 \pm 0.02$ & $2.07 \pm 0.07$ & $0.66 \pm 0.03$ & $0.02 \pm 0.05$ \\
HSE I & $0.53 \pm 0.06^{*}$ & $1.63 \pm 0.17^{*}$ & $0.57 \pm 0.05$ & $0.03 \pm 0.02$ \\
HSE II & $0.44 \pm 0.08^{*}$ & $1.27 \pm 0.25^{*}$ & $0.55 \pm 0.09$ & $-0.02 \pm 0.04$ \\
Contr $\times$ HSE & $0.70 \pm 0.03$ & $2.28 \pm 0.07$ & $0.68 \pm 0.03$ & $-0.05 \pm 0.03$ \\
FL I & $0.53 \pm 0.05^{*}$ & $1.77 \pm 0.15^{*}$ & $0.69 \pm 0.06$ & $-0.02 \pm 0.02$ \\
FL II & $0.56 \pm 0.04^{*}$ & $1.90 \pm 0.13^{*}$ & $0.70 \pm 0.05$ & $-0.07 \pm 0.03$ \\
Contr $\times$ FL & $0.72 \pm 0.02$ & $2.34 \pm 0.05$ & $0.70 \pm 0.03$ & $-0.09 \pm 0.04$ \\
\hline
\end{tabular}

${ }^{*} P<0.05$, compared with the corresponding control group. Patients with HSE showed only a marginally lower $\lambda$ then their controls, $P<0.1$ both at the first and second testing sessions. Some of the measures are also significantly different between control groups (not indicated), as they depend on age and education, which were matched to each patient group. AD, Alzheimer's disease; FL, frontal lobe; HSE, herpes simplex encephalitis. 


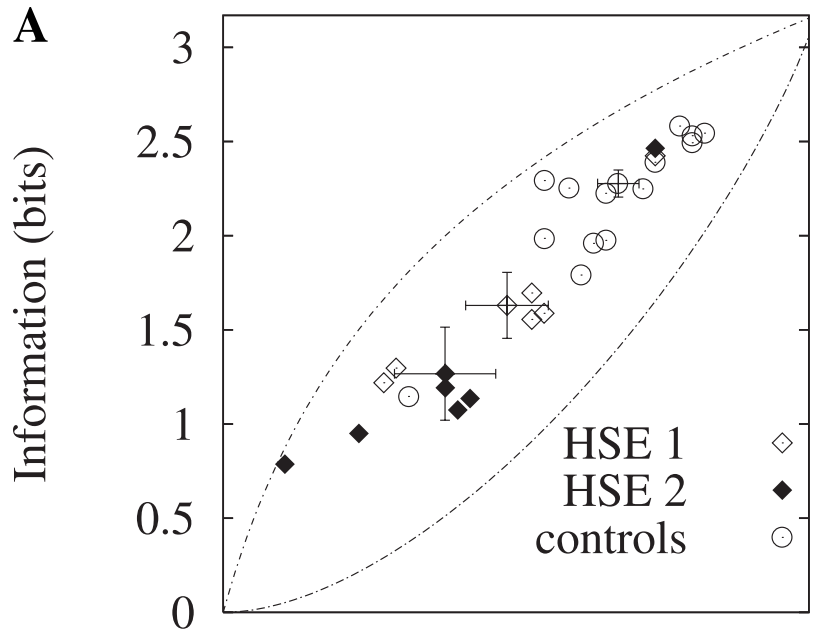

B
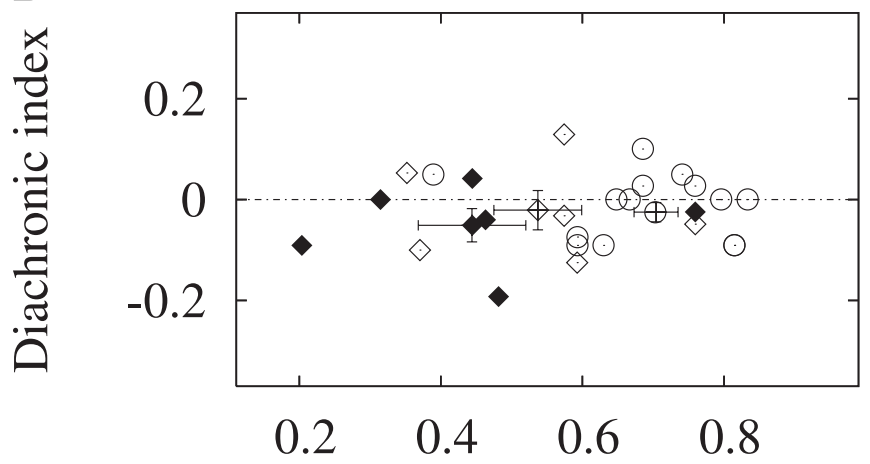

Fraction correct

FIG. 4. (A and B) Performance of herpetic patients and of their controls. Notation as in Fig. 3A and B. HSE, herpes simplex encephalitis.

Indeed, the diachronic index was similar to that of normal controls in both testing sessions ( $P>0.05$ in both cases).

At odds with the patients with $\mathrm{AD}$, patients with HSE showed a marginally lower $\lambda$ then their controls, both at the first ( 0.57 vs 0.68$)$ and the second testing sessions $(0.55$ vs $0.68 ; P<0.1$ in both cases). In parallel, patients showed a lower proportion $f_{\text {p.e. }} / f_{\text {err }}$ with respect to control subjects both at the first $\left(0.66\right.$ vs 0.79 ; Student's $t_{16}=2.2$, $P<0.05)$ and the second session (0.64 vs 0.79 ; Student's $t_{16}=3.2$, $P<0.005)$.

\section{Patients with FL}

Patients with FL (Fig. 5) showed lower $I$ (1.77 vs 2.34; Student's $\left.t_{25}=-4.3, P<0.001\right)$ and $f_{\text {corr }}\left(0.53\right.$ vs 0.72 ; Student's $t_{25}=-4.5$, $P<0.001)$ compared with their controls at the first testing session, and also at the second ( $I$ : 1.90 vs 2.34 ; Student's $t_{25}=-3.6, P<0.01$; $f_{\text {corr }}: 0.56$ vs 0.72 , Student's $t_{25}=-4.5, P<0.001$ ), even though, in contrast to patients with AD and HSE, their performance did not get worse from the first to the second session, in fact it marginally improved. Their impairment in memory for recent relative to remote famous faces was comparable to that of controls, as we did not find any difference in the diachronic index in both sessions $(P>0.05$ in both cases).

The metric content, $\lambda$, was similar between patients with FL and their controls in both sessions $(P>0.1$ in both cases). In contrast,
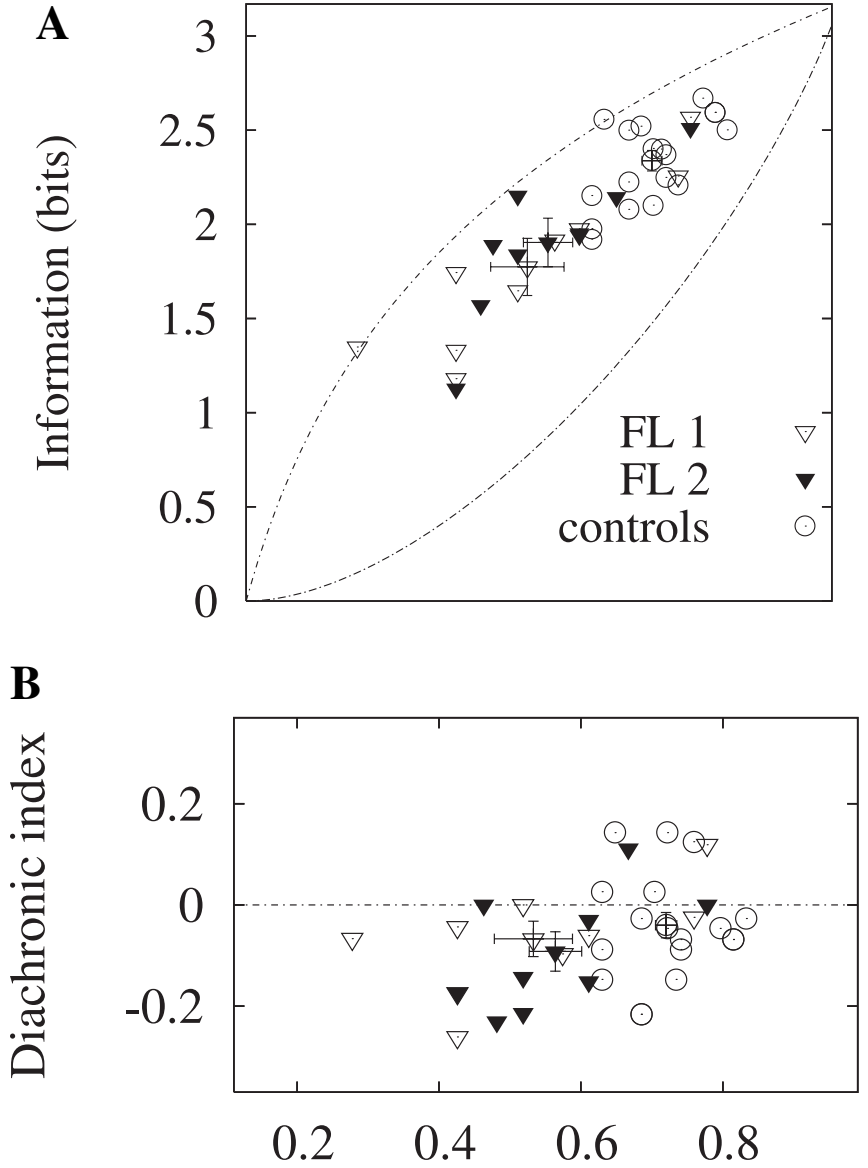

Fraction correct

FIG. 5. (A and B) Performance of (all nine) patients with frontal lobe (FL) and of their controls. Notation as in Fig. 3A and B.

$f_{\text {p.e. }} / f_{\text {err }}$ was significantly lower in patients with FL compared with control subjects $\left(0.57\right.$ vs 0.85 , Student's $\left.t_{25}=-6.5, P<0.0001\right)$. The proportion of partial errors improved significantly in patients with FL from the first to the second testing session ( 0.57 vs 0.73 ; Student's $\left.t_{8}=-3.5, P<0.01\right)$, although it remained lower compared with normal controls $(P<0.001)$.

Similar results were obtained when we limited our analyses to the subset of six prefrontal patients who were homogeneous for aetiology (i.e. ACoA aneurysm), and presented lesions limited to the ventromedial prefrontal cortex. These patients showed lower $I$ (first session: 1.91 vs 2.38 ; Student's $t_{16}=-3.15, P<0.005$; second session: 1.99 vs 2.38; Student's $\left.t_{16}=-3.18, P<0.005\right)$ and $f_{\text {corr }}$ (first session: 0.58 vs 0.72 ; Student's $t_{16}=2.8, P<0.005$; second session: 0.60 vs 0.72 ; Student's $t_{16}=2.9, P<0.005$ ) relative to their 12 age-matched controls. The diachronic index was similar between patients and controls (first session: -0.03 vs -0.02 , $P=0.65$; second session: -0.04 vs $-0.02, P=0.61$ ), as was $\lambda$ (first session: 0.67 vs $0.72, P=0.53$; second session: 0.68 vs 0.72 , $P=0.49)$. In contrast, $f_{\text {p.e. }} / f_{\text {err }}$ was significantly lower in patients with FL compared with control subjects, both at the first $(0.58$ vs 0.87 , Student's $\left.t_{16}=5.07, P<0.0001\right)$ and second sessions $(0.72 \mathrm{vs}$ 0.87 , Student's $\left.t_{16}=-3.5, P<0.001\right)$, even though it improved significantly between sessions $\left(0.58\right.$ vs 0.72 ; Student's $t_{5}=-2.58$, $P<0.05)$. 
As mentioned earlier, a relevant question in the case of patients with FL is whether metric content would correlate with results in executive tests. We found that $\lambda$ was not correlated with either measure of frontal or memory functioning ( $P>0.05$ in all cases). In slight contrast, $f_{\text {p.e. }} / f_{\text {err }}$ showed a trend to correlate with executive functioning in these patients, for example with interference effects in the Stroop task $\left(r_{\text {Pearson }}=-0.5, P<0.15\right)$.

\section{Discussion}

Although there is a general consensus that semantic and episodic memory are distinct, functionally and neurally, little experimental research has been directed at quantifying the separate contributions of these two memory systems to retrograde memory (but see Westmacott et al., 2004; Gilboa et al., 2006). In a previous study (Ciaramelli et al., 2006), we have described an information theoretic approach to quantify the progressive semantization of memories for famous faces with normal ageing, in the absence of specific brain damage. We showed that an index of the concentration of errors, i.e. the metric content index, was higher in older compared with younger adults, thus showing a shift to a preferred semantic access in ageing (Ciaramelli et al., 2006).

In the present study we have explored the effect of brain damage on these memory systems through the same approach, by administering our novel test to patients with $\mathrm{AD}$, patients suffering from HSE, and patients with focal damage in the prefrontal cortex.

All our patient groups were characterized by a decrease in personrelated knowledge compared with their controls, in line with previous evidence (Greene \& Hodges, 1996; Westmacott et al., 2004). However, we found a triple dissociation with respect to metric content in our patient groups, indicating that their preferred access mode was quite different. Patients with $\mathrm{AD}$, usually characterized by a precocious involvement of hippocampal cortices in the neurodegenerative process (e.g. Apostolova et al., 2006; particularly of the CA1 region, Frisoni et al., 2006), showed a marked increase in metric content, indicating a shift to semantic access mode, supporting the notion that episodic access to knowledge, perhaps the default mode (Curran et al., 2006), is mediated by MTL structures, including in particular the hippocampus (see Aggleton \& Brown, 1999; Yonelinas et al., 2005; Gilboa et al., 2006; Moscovitch et al., 2006). The metric content further increased over time, indicating that this measure can track the progress of the disease, which is known to result in progressive hippocampal volume loss and related episodic impairment (Gilboa et al., 2005). Notably, the metric content index appears well suited to capture the nature of the memory changes associated with $\mathrm{AD}$, as the one measure that correlates with measures of the severity of dementia.

To interpret the scattered distribution of patient results, it is useful to consider that the sample was rather heterogeneous with respect to the severity of the degenerative process, and therefore to episodic and possibly semantic memory impairments. Note that the diachronic index $\mathrm{D}$, which in the present study did not differ between patients and controls, in our previous study (Ciaramelli et al., 2006) was not correlated with metric content, so that an increase in the latter is unlikely to result merely from increased semantic cueing of famous faces from the remote past.

In sum, our findings on patients with $\mathrm{AD}$ corroborate extensive evidence from anterograde memory experiments, in which patients with MTL lesions typically give fewer R responses to recognized stimuli than control subjects (Dalla Barba, 1997; Schacter et al., 1997; Yonelinas et al., 1998, 2002), and neuroimaging studies showing a selective involvement of the hippocampus in $\mathrm{R}$ compared with $\mathrm{K}$ responses (Henson et al., 1999; Eldridge et al., 2000; Yonelinas et al.,
2005). Of most relevance for this study, recent fMRI and PET studies have highlighted the role of the hippocampus in accessing retrograde (autobiographic) memory episodically, i.e. re-living specific features of past episodes (Maguire et al., 2003; Piolino et al., 2003; Eustache et al., 2004; Gilboa et al., 2004; Levine et al., 2004; Piolino et al., 2004). Interestingly, the magnitude of hippocampal activity during autobiographical retrieval seems to increase depending on the amount of detail recovered by the subjects: the more vivid the recovered memory, the greater the increase in hippocampal activity (Addis et al., 2004). Moreover, it has been recently demonstrated that whereas retrieval of information regarding celebrities associated with personal memories (Westmacott \& Moscovitch, 2003) activates the hippocampus, this is not observed for equally familiar celebrities not linked to personal memories (Denkova et al., 2006).

We note, however, that the increase in metric content we are seeing in patients with $\mathrm{AD}$ may result not only from a pure 'semantization' of memory, i.e. a shift to a preferred semantic access mode, but also from a progressive loss of subordinate information in semantic memory representations, which is also typical of the disease. Indeed, not only did metric content correlate with performance in an episodic memory test, but also with category fluency, which clearly taps the accessibility of semantic information.

In marked contrast, results with herpetic patients, in the context of a similarly poor long-term memory performance, showed a slight decrease in metric content compared with normal controls. Thus, patients with HSE seem to resort to a preferred episodic access mode to person-related knowledge, which is consistent with the idea that the lateral temporal neocortex, commonly damaged in this pathological condition (e.g. Kapur et al., 1994), contributes to the retrieval of semantic information. Our results on patients with HSE are also in line with evidence from patients with semantic dementia, who show medial-temporal cortex damage typically involving perirhinal (neo)cortex and relatively sparing the hippocampus (Lee et al., 2007). As we discussed earlier, these patients show a preferential sparing of information about personally known individuals relative to equally famous celebrities of no personal significance (Westmacott et al., 2001; see also Snowden et al., 1996) and, compared with patients with $\mathrm{AD}$, of scenes relative to faces (Lee et al., 2007). Taken together, this evidence suggests that person-related knowledge after temporal neocortex damage gets more episodic than semantic in nature (Graham et al., 1999), which is what we observed in patients with HSE. Admittedly, our evidence on HSE is gathered by a small and not quite controlled group of patients, but it constitutes preliminary evidence for the impact of lateral temporal lobe damage on metric content.

Finally, we investigated the pattern of performance sustained by a group of frontal patients in an attempt to sort out the effect of explicit strategic use of cueing information from the effect of implicitly relying on semantic access mode to memory representations. Like patients with $\mathrm{AD}$ and HSE, patients with prefrontal lesions present impaired memory for person-related knowledge. Unlike the other patient groups, however, patients with FL do not show significant changes in their preferred access mode to knowledge as assessed by our metric content index, which takes almost identical values as for their control subjects. Moreover, at the second testing session their overall performance improved, whereas it deteriorated in the other groups.

That the preferred access mode did not change after prefrontal lesions is inconsistent with previous neuropsychological (Wheeler \& Stuss, 2003) and neuroimaging evidence (see Levine et al., 2004; Svoboda et al., 2006 for recent reviews) of impaired recollection after prefrontal cortex lesions. However, other studies have demonstrated 
that patients with lesions in the prefrontal cortex have preserved indicators of recollection (i.e. R responses; Duarte et al., 2005; Ciaramelli \& Ghetti, 2007). It is important to note, also, that to the extent that metric content quantifies the relative contributions to memory retrieval of the hippocampus and temporal neocortex, it is not surprising that it is not impaired in patients with FL, who have no damage in these regions. This reinforces the proposal that memory problems in patients with prefrontal lesions are due to the lack of strategic systems operating on the products of the MTL, rather then on a failure of mnemonic systems per se (see Burgess \& Shallice, 1996; Moscovitch \& Melo, 1997). Consistent with this idea, patients with FL showed a lower proportion of partial errors then did their controls.

In summary, we report that an information theoretic measure of the relative concentration of errors, the metric content index, proved to be effective in differentiating patients with different brain pathology in their preferred access mode to memory. Such a measure was found to track the progression of the disease, and to be less susceptible to the use of deliberate response strategies then previously used measures.

\section{Acknowledgements}

We thank Francesca Borgo for her contribution in testing herpetic patients, and all patients and control subjects for their participation in the study.

\section{Abbreviations}

AD, Alzheimer's disease; FFMCT, Famous Faces Multiple Choice Test; FL, frontal lobe; HSE, herpes simplex encephalitis; MTL, medial-temporal lobe.

\section{References}

Addis, D.R., Moscovitch, M., Crawley, A.P. \& McAndrews, M.P. (2004) Recollective qualities modulate hippocampal activation during autobiographical memory retrieval. Hippocampus, 14, 752-762.

Aggleton, J.P. \& Brown, M.W. (1999) Episodic memory, amnesia, and the hippocampal-anterior thalamic axis. Behav. Brain Sci., 22, 425-444.

Amit, J.D. (1989) Modeling Brain Function. Cambridge UP, Cambridge, UK. Apostolova, L.G., Dinov, I.D., Dutton, R.A., Hayashi, K.M., Toga, A.W., Cummings, J.L. \& Thompson, P.M. (2006) 3D comparison of hippocampal atrophy in amnestic mild cognitive impairment and Alzheimer's disease. Brain, 129, 2867-2873.

Bernard, F.A., Bullmore, E.T., Graham, K.S., Thomson, S.A., Hodges, J.R. \& Fletcher, P.C. (2004) The hippocampal region is involved in successful recognition of both remote and recent famous faces. Neuroimage, 22, 17041714.

Borgo, F. \& Shallice, T. (2001) When living things and other 'sensory quality' categories behave in the same fashion: a novel category specificity effect. Neurocase, 7, 201-220.

Bozeat, S., Lambon Ralph, M.A., Patterson, K., Garrard, P. \& Hodges, J.R. (2000) Non-verbal semantic impairment in semantic dementia. Neuropsychologia, 38, 1207-1215.

Bracco, L., Amaducci, L., Pedone, D., Bino, G., Lazzaro, M.P., Carella, F., D'Antona, Gallato, R. \& Denes, G. (1990) Italian Multicentre Study on Dementia (SMID): a neuropsychological test battery for assessing Alzheimer's disease. J. Psychiat. Res., 24, 213-226.

Braitenberg, V. \& Shüz, A. (1991) Anatomy of the Cortex: Statistics and Geometry. Springer, Berlin.

Burgess, P.W. \& Shallice, T. (1996) Confabulation and the control of recollection. Memory, 4, 359-411.

Carlton, A.G. (1969) On the bias of information estimates. Psych. Bull., 71, 108-109.

Ciaramelli, E. \& Ghetti, S. (2007) What are confabulators' memories made of? A study of subjective and objective measures of recollection in confabulation. Neuropsychologia, 45, 1489-1500.

Ciaramelli, E., Lauro-Grotto, R. \& Treves, A. (2006) Dissociating episodic from semantic access mode by mutual information measures: evidence from aging and Alzheimer's disease. J. Physiol.-Paris, 100, 142-153.
Curran, T., DeBuse, C., Woroch, B. \& Hirshman, E. (2006) Combined pharmacological and electrophysiological dissociation of familiarity and recollection. J. Neurosci., 26, 1979-1985.

Dalla Barba, G. (1997) Recognition memory and recollective experience in Alzheimer's disease. Memory, 5, 657-672.

Denkova, E., Botzung, A. \& Manning, L. (2006) Neural correlates of remembering/knowing famous people: anevent-related fMRI study. Neuropsychologia, 44, 2783-2791.

Duarte, A., Ranganath, C. \& Knight, R.T. (2005) Effects of unilateral prefrontal lesions on familiarity, recollection, and source memory. J. Neurosci., 25, 8333-8337.

Eichenbaum, H. (2006) Remembering: functional organization of the declarative memory system. Curr. Biol., 16, 643-645.

Eldridge, L.L., Knowlton, B.J., Furmanski, C.S., Bookheimer, S.Y. \& Engel, S.A. (2000) Remembering episodes: a selective role for the hippocampus during retrieval. Nat. Neurosci., 3, 1149-1153.

Eustache, F., Piolino, P., Giffard, B., Viader, F., de la Sayette, V., Baron, J.C. \& Desgranges, B. (2004) 'In the course of time': a PET study of the cerebral substrates of autobiographical amnesia in Alzheimer's disease. Brain, 127, $1549-1560$.

Folstein, M.F., Folstein, S.E. \& McHugh, P.R. (1975) 'Mini Mental State': a practical method for grading the cognitive state of patients for the clinician. J. Psychiat. Res., 12, 189-198.

Frisoni, G.B., Sabattoli, F., Lee, A.D., Dutton, R.A., Toga, A.W. \& Thompson, P.M. (2006) In vivo neuropathology of the hippocampal formation in AD: a radial mapping MR-based study. Neuroimage, 32, 104-110.

Gilboa, A. (2004) Autobiographical and episodic memory - one and the same? Evidence from prefrontal activation in neuroimaging studies. Neuropsychologia, 42, 1336-1349.

Gilboa, A., Ramirez, J., Kohler, S., Westmacott, R., Black, S.E. \& Moscovitch, M. (2005) Retrieval of autobiographical memory in Alzheimer's disease: relation to volumes of medial temporal lobe and other structures. Hippocampus, 15, 535-550.

Gilboa, A., Winocur, G., Grady, C.L., Hevenor, S.J. \& Moscovitch, M. (2004) Remembering our past: functional neuroanatomy of recollection of recent and very remote personal events. Cereb. Cortex, 14, 1214-1225.

Gilboa, A., Winocur, G., Rosenbaum, S.R., Poreh, A., Gao, F., Black, S.E., Westmacott, R. \& Moscovitch, M. (2006) Hippocampal contributions to recollection in retrograde and anterograde amnesia. Hippocampus, 16, 966980.

Graham, K.S. \& Hodges, J.R. (1997) Differentiating the roles of the hippocampal complex and the neocortex in long-term memory storage: evidence from the study of semantic dementia and Alzheimer's disease. Neuropsychology, 11, 77-89.

Graham, K.S., Patterson, K. \& Hodges, J.R. (1999) Episodic memory: new insights from the study of semantic dementia. Curr. Opin. Neurobiol., 9, $245-250$

Greene, J.D. \& Hodges, J.R. (1996) The fractionation of remote memory. Evidence from a longitudinal study of dementia of Alzheimer type. Brain, 119, 129-142.

Henson, R.N., Rugg, M.D., Shallice, T., Josephs, O. \& Dolan, R.J. (1999) Recollection and familiarity in recognition memory: an event-related functional magnetic resonance imaging study. J. Neurosci., 19, 39623972 .

Isaacs, B. \& Kennie, A. (1973) The Set Test as an aid to the detection of dementia in old people. Br. J. Psychiatry, 123, 467-470.

Kapur, N., Barker, S., Burrows, E.K., Ellison, D., Brice, J., Illis, L.S., Scholey, K., Colbourn, C., Wilson, B. \& Loates, M. (1994) Herpes simplex encephalitis: long term magnetic resonance imaging and neuropsychological profile. J. Neurol. Neurosurg. Psychiatry, 57, 1334-1342.

Kropff, E. \& Treves, A. (2007) Uninformative memories will prevail: effective storage of correlated representations and its consequences. HFSP Journal 1, doi: $10.2976 / 1.2793335$.

Lauro-Grotto, R., Piccini, C. \& Shallice, T. (1997) Modality specific operations in semantic dementia. Cortex, 33, 593-622.

Lee, A.C.H., Levi, N., Davies, R.R., Hodges, J.R. \& Graham, K.S. (2007) Differing profiles of face and scene discrimination deficits in semantic dementia and Alzheimer's disease. Neuropsychologia, 45, 21352146.

Leutgeb, J.K., Leutgeb, S., Moser, M.B. \& Moser, E.I. (2007) Pattern separation in the dentate gyrus and CA3 of the hippocampus. Science, $\mathbf{3 1 5}$, 961-966.

Leutgeb, S., Leutgeb, J.K., Treves, A., Moser, M.B. \& Moser, E.I. (2004) Distinct ensemble codes in hippocampal areas CA3 and CA1. Science, $\mathbf{3 0 5}$, $1295-1298$. 
Levine, B., Turner, G.R., Tisserand, D.J., Hevenor, S.J., Graham, S.J. \& McIntosh, A.R. (2004) The functional neuroanatomy of episodic and semantic remembering: a prospective functional MRI study. J. Cogn. Neurosci., 16, 1633-1646.

Maguire, E.A., Valentine, E.R., Wilding, J.M. \& Kapur, N. (2003) Routes to remembering: the brains behind superior memory. Nat. Neurosci., 6, 90-95.

Marr, D. (1971) Simple memory: a theory for archicortex. Phil. Trans. R. Soc. Lond., 262 (Series B), 23-81.

McNaughton, B.L. \& Morris, R.G.M. (1987) Hippocampal synaptic enhancement and information storage within a distributed memory system. Trends Neurosci., 10, 408-415.

Moscovitch, M. \& Melo, B. (1997) Strategic retrieval and the frontal lobes: evidence from confabulation and amnesia. Neuropsychologia, 35, 10171034.

Moscovitch, M., Nadel, L., Winocur, G., Gilboa, A. \& Rosenbaum, R.S. (2006) The cognitive neuroscience of remote episodic, semantic and spatial memory. Curr. Opin. Neurobiol., 16, 179-190.

Panzeri, S. \& Treves, A. (1996) Analytical estimates of limited sampling biases in different information measures. Network, 7, 87-107.

Piolino, P., Desgranges, B., Belliard, D., Matuszewski, V., Lavalee, C., de la Sayette, V. \& Eustache, F. (2003) Episodic memory and autonoetic awareness: triple dissociation in neurodegenerative diseases. Brain, 126, 2203-2219.

Piolino, P., Giffard-Quillon, G., Desgranges, B., Chetelat, G., Baron, J.C. \& Eustache, F. (2004) Re-experiencing old memories via hippocampus: a PET study of autobiographical memory. Neuroimage, 22, 13711383.

Rolls, E.T. (1989) Functions of neuronal networks in the hippocampus and cerebral cortex in memory. In Cotterill, R. (Ed.), Models of Brain Function. Cambridge UP, Cambridge, UK, pp. 15-33.

Rolls, E.T. (2000) Hippocampo-cortical and cortico-cortical backprojections. Hippocampus, 10, 380-388.

Schacter, D.L., Verfaellie, M. \& Anes, M.D. (1997) Illusory memories in amnesic patients: conceptual and perceptual false recognition. Neuropsychology, 11, 331-342.

Seidenberg, M., Griffith, R., Sabsevitz, D., Moran, M., Haltiner, A., Bell, B., Swanson, S., Hammeke, T. \& Hermann, B. (2002) Recognition and identification of famous faces in patients with unilateral temporal lobe epilepsy. Neuropsychologia, 40, 446-456.

Snowden, J.S., Griffiths, H.L. \& Neary, D. (1994) Semantic dementia: autobiographical contribution to preservation of meaning. Cogn. Neuropsychol., 11, 265-288.

Snowden, J.S., Griffiths, H.L. \& Neary, D. (1996) Autobiographical experience and word meaning. Memory, 3, 225-246.
Spinnler, H. \& Tognoni, G. (1987) Standardizzazione e Taratura Italiana di Test Neuropsicologici. Ital. J. Neurol. Sci., 6 (Suppl. 8), 1-120.

Svoboda, E., McKinnon, M.C. \& Levine, B. (2006) The functional neuroanatomy of autobiographical memory: a meta-analysis. Neuropsychologia, 44, 2189-2208.

Treves, A. (1997) On the perceptual structure of face space. Biosystems, 40, 189-196.

Treves, A. \& Rolls, E.T. (1992) Computational constraints suggest the need for two distinct input systems to the hippocampal CA3 network. Hippocampus, 2, 189-199.

Treves, A. \& Rolls, E.T. (1994) Computational analysis of the role of the hippocampus in memory. Hippocampus, 4, 374-391.

Tulving, E. (1985) Memory and consciousness. Can. Psychol., 26, 1-12.

Tulving, E. (2002) Episodic memory: from mind to brain. Annu. Rev. Psychol., 53, 1-25.

Vazdarjanova, A. \& Guzowski, J.F. (2004) Differences in hippocampal neuronal population responses to modifications of an environmental context: evidence for distinct, yet complementary, functions of CA3 and CA1 ensembles. J. Neurosci., 24, 6489-6496.

Westmacott, R., Black, S.E., Freedman, M. \& Moscovitch, M. (2004) The contribution of autobiographical significance to semantic memory: evidence from Alzheimer's disease, semantic dementia, and amnesia. Neuropsychologia, 42, 25-48.

Westmacott, R., Leach, L., Morris, F. \& Moscovitch, M. (2001) Different patterns of autobiographical memory loss in semantic dementia and medial temporal lobe amnesia: a challenge to consolidation theory. Neurocase, 7 , 37-55.

Westmacott, R. \& Moscovitch, M. (2003) The contribution of autobiographical significance to semantic memory. Mem. Cogn., 31, 761-774.

Wheeler, M.E. \& Buckner, R.L. (2004) Functional-anatomic correlates of remembering and knowing. Neuroimage, 21, 1337-1349.

Wheeler, M.A. \& Stuss, D.T. (2003) Remembering and knowing in patients with frontal lobe injuries. Cortex, 39, 827-846.

Yonelinas, A.P., Kroll, N.E., Dobbins, I., Lazzara, M. \& Knight, R.T. (1998) Recollection and familiarity deficits in amnesia: convergence of remember/know, process dissociation, and receiver operating characteristic data. Neuropsychology, 12, 323-339.

Yonelinas, A.P., Kroll, N.E., Quamme, J.R., Lazzara, M.M., Sauve, M.J., Widaman, K.F. \& Knight, R.T. (2002) Effects of extensive temporal lobe damage or mild hypoxia on recollection and familiarity. Nat. Neurosci., 5 , 1236-1241.

Yonelinas, A.P., Otten, L.J., Shaw, K.N. \& Rugg, M.D. (2005) Separating the brain regions involved in recollection and familiarity in recognition memory. J. Neurosci., 16, 3002-3008. 